\title{
Experiencias de desempeño y creencias de autoeficacia en profesores de lenguas extranjeras
}

\author{
Enactive mastery experiences and the self-efficacy \\ beliefs of foreign language teachers
}

Griselda MuRRIETA LOYO*

María del Rosario Reyes Cruz ${ }^{* *}$

El objetivo de nuestro trabajo es explorar las experiencias de desempeño de siete profesores de lenguas extranjeras a lo largo de su trayectoria y la relación que estas guardan con sus creencias de autoeficacia docente. Utilizamos la teoría de la autoeficacia de Bandura (1997) y el diseño cualitativo de tipo exploratorio; realizamos entrevistas en profundidad a siete profesores experimentados de una universidad pública mexicana. Encontramos que, al inicio de su trayectoria, los profesores no se sentían muy eficaces al impartir clases por la falta de práctica real durante su formación. Los profesores emplearon la reflexión, la formación y el esfuerzo como vías para mejorar su eficacia percibida. Su experiencia más extensa ha sido enseñando idiomas extranjeros en un centro de lenguas universitario. Concluimos que la experiencia previa sí influye en las creencias de autoeficacia de los profesores y que estas se diferencian según el tipo de materia y nivel educativo que enseñan. El dominio del idioma también apareció como un factor influyente en las creencias de autoeficacia.

The objective of this study was to explore the enactive mastery experiences that seven foreign language teachers have had throughout their academic trajectory and the relation they have with their self-efficacy beliefs in teaching. Bandura's theory (1997) on self-efficacy was used as a theoretical approach. The design used was qualitative of exploratory type; in-depth interviews were conducted with 7 experienced teachers from a Mexican public university. It was found that at the beginning of their trajectory, teachers did not perceive themselves as very effective to teach due to the lack of real practice during their teacher training. Teachers used reflection, training and effort as ways to improve their perceived self-efficacy. The most extensive experience of these teachers has been teaching foreign languages in a university language center. It is concluded that previous experience does influence teachers' self-efficacy beliefs and that these beliefs differ according to the type of subject and the educational level at which they teach. Language proficiency also proved to be an influential factor in self-efficacy beliefs.

\author{
Palabras clave: \\ creencias de \\ autoeficacia, \\ experiencias de \\ desempeño, \\ lenguas \\ extranjeras, \\ profesores
}

Recibido: 12 de noviembre de 2018. | Aceptado para su publicación: 29 de abril de 2019. Recuperado de: https://sinectica.iteso.mx/index.php/SINECTICA/article/view/924 DOI: $10.31391 /$ S2007-7033(2019)0052-010

\footnotetext{
* Doctora en Educación. Profesora-investigadora de la Universidad de Quintana Roo. Líneas de investigación: el estado del arte de la investigación en la enseñanza de lenguas extranjeras, uso y acceso a la tecnología educativa, creencias epistemológicas en la enseñanza y el aprendizaje de lenguas extranjeras, creencias de autoeficacia en la enseñanza y el aprendizaje de lenguas extranjeras. Correo electrónico: grismurrieta@hotmail.com

${ }^{* *}$ Doctora en Educación Internacional. Profesora-investigadora de la Universidad de Quintana Roo. Miembro del Sistema Nacional de Investigadores. Líneas de investigación: tecnologías de la información y la comunicación, creencias de autoeficacia en la docencia y el aprendizaje de lenguas extranjeras. Correo electrónico: rosreyescruz@hotmail.com
} 


\section{INTRODUCCIÓN}

T a investigación internacional ha mostrado que las creencias de autoeficacia en los profesores de lenguas se relacionan con el nivel de dominio del idioma enseñado (Marashi \& Azizi-Nassab, 2018), con la expresión oral (Mirsanjari, Karbalaei \& Afraz, 2013) y con las habilidades de instrucción (Goker, 2006), entre otras. No obstante, en México este tema ha sido poco explorado, y solo identificamos los trabajos de Cocca y Cocca (2016), Cocca, Cocca, Alvarado y Rodríguez (2018) y Reyes Murrieta (2017).

La globalización ha generado la necesidad de desarrollar habilidades para comunicarse en lenguas diferentes de la materna (Díez, 2014). En esa línea de pensamiento, la Secretaría de Educación Pública (2008) estima que la enseñanza de una lengua extranjera reviste gran importancia en el perfil del futuro profesionista mexicano. Por tal razón, la figura del profesor es en especial relevante puesto que es el encargado de desarrollar las habilidades lingüísticas de quienes se insertarán en un mercado laboral donde el dominio de lenguas extranjeras es altamente valorado. Los profesores de idiomas deben, entonces, contar con una buena formación (O’Donoghue, 2015) y con creencias de autoeficacia docente adecuadas que les permitan influir de manera positiva en sus estudiantes.

Es importante mencionar que en México por décadas la enseñanza de idiomas la realizaban personas que "caían en la profesión", normalmente nativos hablantes (Lengeling, 2010), sin título de licenciatura o formados en otras profesiones. Existían pocas licenciaturas y los profesores impartían clases al público en general en centros de idiomas. La Secretaría de Educación Pública puso en marcha, en 1996, el Programa de Mejoramiento del Profesorado, cuyo objetivo era profesionalizar a los docentes y mejorar su habilitación académica. En ese contexto, muchos de los profesores de lenguas tuvieron la posibilidad de obtener grados académicos superiores. Los profesores, al titularse de sus posgrados, comenzaron a la vez a diseñar y ofrecer varias maestrías a lo largo del país.

De lo anterior se deriva que la identidad del profesor de lenguas durante muchos años haya sido el de una persona que domina un idioma y lo enseña; podemos intuir que el nivel de lengua era su principal fuente de eficacia personal. No obstante, los profesores han experimentado alteraciones en su identidad debido a que las políticas educativas actuales y el contexto de enseñanza los han empujado a impartir materias de contenido en licenciatura y posgrado. Es lógico pensar que, en estas actividades relativamente recientes, no exista aun una identidad estable y, por ende, el sentido de autoeficacia sea variable y se encuentre menos establecido con firmeza. El sentido de autoeficacia y la identidad se afectan de manera mutua y, a la vez, influyen en el desempeño del profesor (Ghafar, Kiany, Akbari \& Azimi, 2011).

La importancia actual de dominar lenguas y la influencia de las creencias de autoeficacia en diferentes esferas de la enseñanza invitan a realizar estudios que sumen conocimiento empírico a lo poco que se conoce sobre este tema en México. En esa línea de pensamiento, el objetivo de este trabajo fue explorar las experiencias de desempeño que los profesores de lenguas extranjeras de un centro de lenguas han tenido a lo largo de su trayectoria y la relación que estas guardan con sus creencias de autoeficacia docente. 


\section{LAS CREENCIAS DE AUTOEFICACIA Y LA DOCENCIA}

En esta investigación se define el sentido de autoeficacia para la docencia como "la creencia de los profesores en su capacidad para organizar y ejecutar las acciones necesarias para llevar a cabo de manera exitosa tareas específicas de enseñanza en un contexto determinado" (Tschannen-Moran \& Hoy, 2001, p. 796). Según Bandura (1997), la enseñanza es una actividad compleja, una construcción organizada y controlada, en su mayoría, por habilidades cognitivas y autorregulatorias. El conocimiento de las reglas y estrategias de la docencia para determinar cursos de acción permite a los profesores manejar las exigencias de su quehacer diario.

Según Bandura (1997), existen cuatro fuentes de información que contribuyen a la formación de las creencias de autoeficacia: experiencias reales de desempeño, experiencias vicarias, persuasión verbal y estados fisiológicos y emocionales. Bandura (1997) considera las primeras como las más influyentes, porque permiten al individuo darse cuenta de sus logros, dificultades o fracasos en el desempeño de la actividad real. Debido a lo anterior, a la extensión y complejidad que amerita su estudio, en este trabajo nos centramos únicamente en las experiencias de desempeño como fuente de las creencias de autoeficacia docente.

Bandura (1997) sostiene que la forma de evaluar las experiencias previas de desempeño y, por ende, la eficacia personal influye en varios aspectos. Uno de ellos son las estructuras preexistentes de autoconocimiento; es decir, la persona alberga creencias sobre sí misma basadas en sus experiencias pasadas. Aquellas que no son consistentes con las creencias ya establecidas tienden a excluirse, minimizarse $u$ olvidarse. Por el contrario, las que son congruentes, en seguida se perciben, se les atribuye sentido y se recuerdan con facilidad (Bandura, 1997, p. 82).

El grado de esfuerzo que pongan los individuos en las actividades que realizan interviene en las inferencias que hacen sobre su desempeño en ellas (Bandura, 1997). Estas inferencias difieren de persona a persona: en algunos casos se cree que una alta inversión de esfuerzo es muestra de una baja capacidad. No obstante, otros estudios han encontrado que el esfuerzo mejora la habilidad (Sun, Chen \& Song, 2016). El automonitoreo selectivo también interviene en la reconstrucción de las experiencias de desempeño. Los desempeños suceden en situaciones y contextos muy variados, por lo cual no siempre se puede tener control de ellos. Los individuos difieren en la forma en que observan y recuerdan su actuación.

Bandura (1997) concluye que la mayoría de las competencias complejas, como es el caso de la docencia, requiere una serie de subhabilidades que deben organizarse e integrarse de manera jerárquica en condiciones fluctuantes por un largo periodo; es decir, toma tiempo convertirse en un profesor eficaz. Finalmente, la manera en que se evalúa la eficacia en los desempeños previos depende de su representación cognitiva y de la forma en que se recuerdan. Esta evaluación no es necesariamente verdadera; es la percepción personal del individuo.

El modelo más influyente de la aplicación de la teoría de la autoeficacia en la docencia en general es el de Tschannen-Moran, Woolfolk y Hoy (1998). Estos autores adicionan al modelo de Bandura la evaluación por parte de los profesores de su propia competencia para enseñar en un contexto determinado, y toman en cuenta los 
requisitos de la actividad específica de enseñanza y una evaluación de su competencia personal en vista de tales requisitos.

Palmer (2006) argumenta que en los profesores en formación podrían existir dos tipos más de experiencias de desempeño: dominio de contenido (conocimiento de un cierto tema) y dominio cognitivo-pedagógico (dominio de la comprensión de conocimiento pedagógico especializado). En el caso de los profesores de lenguas extranjeras no existe, hasta donde sabemos, un modelo específico sobre cómo se comportan las creencias de autoeficacia y sus fuentes. Aunque vale la pena mencionar que el nivel de dominio de lengua sobresale como un aspecto que influye particularmente en las creencias de autoeficacia de los profesores de esta área (Best, 2014; Marashi \& Azizi-Nassab, 2018; Slami \& Fatahi, 2008).

Existen pocos trabajos que hayan investigado en específico la relación entre las experiencias reales de desempeño y las creencias de autoeficacia docente en profesores de lenguas en servicio. Gran parte de estos han encontrado que, a mayor experiencia, mayor sentido de autoeficacia (Afshar, Rahimi, Ghonchehpour \& Saedpanah, 2015; Cocca \& Cocca, 2016; Morris \& Usher, 2011; Praver, 2014). No obstante, los trabajos en educación han tenido resultados divergentes. Hoy y Spero (2005) descubrieron que el sentido de autoeficacia se incrementa durante el periodo de preparación de los profesores, pero baja durante el primer año de enseñanza. Por otra parte, Tschannen-Moran y Hoy (2007) hallaron que los factores contextuales juegan un papel preponderante en el sentido de autoeficacia de los profesores principiantes, mientras que la experiencia previa tiene un rol secundario. Klassen y Chiu (2010) mostraron que el sentido de autoeficacia aumentaba de modo progresivo hasta los veintitrés años de experiencia docente y luego disminuía.

\section{MÉTODO}

Este estudio cualitativo de tipo exploratorio (Singh, 2007) busca añadir conocimiento empírico acerca de las creencias de autoeficacia en la docencia de los profesores de lenguas extranjeras, tema escasamente explorado en México y en Latinoamérica en general. Sumar información sobre el particular permitirá contar con mayores elementos para explicar cómo se da el fenómeno en este país.

\section{Contexto}

Esta investigación se realizó en la Universidad de Quintana Roo, institución que cuenta con un centro de lenguas que imparte cursos de diferentes idiomas extranjeros (inglés, francés, alemán, italiano), una lengua originaria (maya) y español como lengua extranjera. El público principal son los estudiantes de las diversas licenciaturas que ofrece la Universidad, dado que estos deben alcanzar cierto nivel de inglés como requisito para titularse. Además, toman clases allí profesores y personal administrativo. También asisten personas externas a la institución, provenientes de diferentes niveles educativos. Asimismo, en la Universidad se imparte una licenciatura para formar profesores de inglés y una maestría en Educación. 


\section{Muestra}

La selección de la muestra fue por conveniencia y por criterio (Creswell, 2007). El criterio consistió en que los participantes fueran profesores de tiempo completo (PTC), ya que en el mismo centro también laboran profesores por asignatura. Ocho profesores cumplían con el criterio, pero únicamente siete (seis mujeres y un hombre) estuvieron dispuestos a dar entrevista. Todos los profesores cuentan con una maestría en Educación como grado máximo y el nivel C1 de certificación en francés o en inglés, según sea el idioma que enseñen.

Todos los participantes han recibido entre diez y quince cursos en diversas áreas de formación docente en la enseñanza de lenguas, como evaluación, competencias, didáctica y técnicas de enseñanza de lenguas. Además de estos cursos en común, algunos profesores han tomado otros sobre tutorías (participantes 1, 6 y 3), investigación (participantes 2 y 6) y tecnología educativa (participantes 5, 7 y 4). Las profesoras de francés han tomado diplomados en formación de asesores para centros de autoacceso y cursos para ser evaluadores de los exámenes de certificación DELF de francés. El participante 7 cursó dos especialidades: una en diseño de proyectos didácticos y otra en diseño de programas de estudio por competencias. En la tabla 1 puede observarse el número asignado a los participantes, su información demográfica y sus años de experiencia totales.

Tabla 1. Información de los participantes

\begin{tabular}{|c|c|c|c|c|c|}
\hline Participante & Sexo & Edad & Lengua impartida & $\begin{array}{c}\text { Año de ingreso } \\
\text { como PTC }\end{array}$ & $\begin{array}{c}\text { Total de años } \\
\text { de experiencia }\end{array}$ \\
\hline 1 & Femenino & 44 & Francés & 2005 & 17.5 \\
\hline 2 & Femenino & 36 & Inglés & 2008 & 14 \\
\hline 3 & Femenino & 42 & Inglés & 2000 & 19 \\
\hline 4 & Femenino & 45 & Francés & 2002 & 19 \\
\hline 5 & Femenino & 43 & Inglés, francés & 2002 & 20 \\
\hline 6 & Femenino & 43 & Inglés & 2002 & 17 \\
\hline 7 & Masculino & 31 & Inglés & 2016 & 16 \\
\hline
\end{tabular}

\section{Instrumento}

Se empleó una guía de entrevista semiestructurada (Hernández, Collado y Baptista, 2006) cuyas preguntas versaban sobre las experiencias reales de desempeño que han tenido los profesores, su sentido de autoeficacia para enseñar una lengua extranjera, los elementos que influyen en esta percepción y los cambios que han experimentado a lo largo de los años. Las dimensiones de las que partimos fueron establecidas con base en la teoría de la autoeficacia y en los objetivos de la investigación.

\section{Procedimiento}

Invitamos a los profesores a participar en el estudio, les explicamos el objetivo de la investigación y les solicitamos que firmaran un consentimiento informado. A lo largo de un mes, realizamos dos entrevistas a cada profesor debido al número de preguntas que contenía la guía y a su disponibilidad de tiempo. La primera entrevista duró en promedio ochenta minutos y la segunda, setenta. Hubo necesidad de una tercera entrevista a algunos profesores para esclarecer dudas e imprecisiones. 
Las entrevistas fueron transcritas con la ayuda del programa Dragon Naturally Speaking® y luego las codificamos. Hallamos códigos relacionados con las categorías teóricamente preestablecidas (experiencias previas y creencia de autoeficacia); además, surgieron dos códigos nuevos: el esfuerzo y el nivel de lengua. Realizamos una segunda codificación para refinar la primera y organizamos los códigos en categorías. La validación del análisis se hizo mediante la doble codificación (Hernández, Collado y Baptista, 2006). Ambas coautoras codificamos por separado los datos para después comparar los resultados y tomar decisiones al respecto. De igual forma, co-construimos con los participantes la interpretación de los aspectos cuyo significado no nos quedaba claro (Brown \& Coombe, 2015). De igual modo, revisamos los currículos de los profesores para constatar los años de experiencia, lugar de trabajo, formación y cursos de actualización. También, consultamos los planes de estudios de las licenciaturas y las maestrías cursadas para determinar la consistencia de los datos referentes a la formación docente recibida.

\section{RESULTADOS Y DISCUSIÓN}

\section{Las experiencias como profesores en formación}

Para establecer las experiencias de desempeño de los profesores, desde el inicio hasta la actualidad, comenzamos por explorar la formación recibida en la licenciatura y las oportunidades que tuvieron para enseñar. La mayoría de los profesores comentaron haber recibido formación teórica respecto de la enseñanza, pero no haber hecho prácticas: “Como que más bien toda mi formación fue teórica, qué hacer, cómo hacerlo, pero jamás fue llevado a la práctica” (participante 4).

Muy pocos profesores llevaron a cabo microenseñanza: "Di clases en mi propia universidad, pero a mis mismos compañeros y pues ellos pretendían [fingían] ser estudiantes. Fue una vez, pero [los maestros] no dieron retroalimentación. Ya después me di cuenta que no era lo que yo pensaba, pensaba que sí podía, pero porque mis compañeros me ayudaron a que saliera" (participante 2). Solo una de las profesoras asistió, como parte de su servicio social, en sus clases a un profesor en el Programa Nacional de Inglés en Educación Básica. Estas escasas experiencias, básicamente en contextos ficticios en la etapa de formación, propiciaron que los participantes se sintieran poco eficaces para iniciar su carrera profesional: "Sí, cuando empecé, me ofrecieron una materia y fue la muerte porque dije: ‘Cómo yo voy a poder dar esa materia?', sentía que no sabía" (participante 1).

Como profesores noveles, los participantes desarrollaron conocimientos teóricos sobre los temas y dominio de un área pedagógica especializada (Palmer, 2006), pero no contaban con experiencias de desempeño reales. En la época en la que estos profesores cursaron sus licenciaturas no se ponía particular interés en vincular teoría y práctica; de hecho, se decía que "uno empezaba a aprender después de que salía de la universidad". Actualmente, esa tendencia ha cambiado; sin embargo, para los participantes de este estudio la falta de experiencia en aulas reales constituye una seria desventaja. La docencia implica el despliegue simultáneo de varias habilidades: dominio de contenido, control de grupo, estrategias didácticas, volumen y tono de voz, entre otras. El haber impartido clases a grupos reales pudo haber proporcionado a 
los principiantes la información necesaria para evaluar su capacidad docente (Bandura, 1997) y, en función de ello, emprender estrategias de mejora.

\section{Experiencias de desempeño iniciales}

Los profesores iniciaron su práctica profesional impartiendo clases de inglés, algunos de ellos en diversos niveles educativos al mismo tiempo. La duración de esta actividad se ubica en un rango de entre dos y seis años. Uno de los participantes trabajó como profesor en el Instituto Nacional para la Educación de los Adultos (INEA) durante ocho años; después también lo hizo en otros niveles educativos, pero conservó un tiempo su trabajo inicial. Posteriormente, se desempeñó durante cinco años como responsable de diseño curricular en un departamento de innovación educativa universitario, al mismo tiempo que impartía cursos en licenciaturas y posgrados en diversas instituciones. Por lo anterior, en esos casos los años totales no resultan de la suma de los años en cada nivel. En la tabla 2 se consignan los detalles (los números se refieren a años; en el caso de los meses se explica).

Tabla 2. Experiencias de desempeño iniciales

\begin{tabular}{|c|c|c|c|c|c|c|c|c|c|}
\hline $\mathrm{P}$ & Preparatoria & Secundaria & Primaria & PE & ELEF & INEA & Lic./Mtría. & DIE & Total \\
\hline 1 & 6 meses & 3 & 0 & 0 & 3 & 0 & 0 & 0 & 6.5 \\
\hline 2 & 4 & 6 & 0 & 0 & 0 & 0 & 0 & 0 & 6 \\
\hline 3 & 1 & 0 & 1 & 1 & 0 & 0 & 0 & 0 & 3 \\
\hline 4 & 0 & 1 & 0 & 0 & 3 & 0 & 0 & 0 & 5 \\
\hline 5 & 3 & 0 & 0 & 0 & 3 & 0 & 0 & 0 & 6 \\
\hline 6 & 1 & 1 & 1 & 0 & 0 & 0 & 0 & 0 & 3 \\
\hline 7 & 1 & 6 meses & 1 & 0 & 0 & 8 & 7 & 5 & 15 \\
\hline
\end{tabular}

$\mathrm{P}=$ Participante

ELEF = Español lengua extranjera en Francia

$\mathrm{PE}=$ Preescolar

Lic. $=$ Licenciatura

Mtría. = Maestría

DIE $=$ Departamento de Innovación Educativa

Todos los participantes manifestaron que al inicio de su actividad profesional enfrentaron problemas en el aula de diversa naturaleza, debido, principalmente, a la ausencia de experiencias reales de desempeño en su formación en licenciatura. La participante 6, por ejemplo, mencionó:

Como que me di cuenta que tenía más debilidades de las que creía y eso como que te hace reflexionar, ¿no?, te sacude y tienes que ponerte a trabajar en lo que te hace falta. 
Traté de identificar qué estaba fallando, si era teoría, o estrategias, o cuestiones pedagógicas, o de dinámicas. Entonces, traté de fortalecerme para mejorar en mis clases.

Los problemas que enfrentaron los profesores, en vez de desanimarlos, los alentaron a tomar decisiones para percibirse más eficaces. Entendieron que la diferencia entre su capacidad docente de aquel momento y el nivel que debían lograr para considerarse buenos profesores era posible de subsanar mediante la actualización docente, el automonitoreo de sus carencias y la autorreflexión. Bandura (1977) menciona que cuando las personas perciben discrepancias entre su desempeño y los estándares establecidos se crea una insatisfacción que, normalmente, motiva cambios correctivos en la conducta. Los participantes empezaron a percibirse poco a poco más eficaces, lo que, a la par, los condujo a volverse más estratégicos y, por ende, a mejorar sus creencias de eficacia y crear, así, un círculo virtuoso.

Solo una de las profesoras comentó que se dio cuenta de que era muy difícil enseñar a niños de secundaria y decidió dejar ese trabajo: "Sí, me llevó adaptarme, yo ya no quería hacerlo. Ya no soportaba a esos niños, y lo dejé" (participante 2). El desempeño de esta profesora se vio mermado por las difíciles condiciones que caracterizan la enseñanza del inglés en secundaria en México, como la falta de formación para enseñar en este nivel, la escasez de materiales didácticos y los grupos numerosos. Las circunstancias adversas y la poca experiencia previa hicieron que esta profesora tomara esta experiencia como un fracaso y, finalmente, abandonara este trabajo. Al respecto, Bandura (1997) señala que cuando las personas piensan que han llegado al límite de su capacidad en una determinada actividad tienden a desistir.

También, observamos que las profesoras de francés son las únicas que realizaron una estancia en el extranjero en esta etapa inicial. Esta experiencia les permitió fortalecer sus habilidades lingüísticas y sentirse más eficaces para enseñar este idioma debido al alto dominio logrado. La participante 5 mencionó: "Yo considero que mi habilidad de speaking en francés es mejor que en inglés. Haber vivido en Francia me dio mayor seguridad para enseñarlo".

Las profesoras indicaron que esta experiencia las llevó a concebir la enseñanza del francés como una actividad intercultural: "Yo les enseño que no es solamente aprender el idioma, sino que además voy a hablar con el otro que es diferente a mí. No es solo una cuestión lingüística, sino cultural" (participante 4). Con el auge de las tecnologías basadas en computadora y la promoción institucional de la movilidad estudiantil, las posibilidades de comunicarse con otras culturas han aumentado de manera exponencial. Luego entonces, la capacidad de enseñar lenguas con un enfoque intercultural es considerada una característica del profesor eficaz. Las profesoras en cuestión responden a esta demanda y ello fortalece sus creencias de autoeficacia docente.

Finalmente, destaca la experiencia de ocho años de uno de los participantes como profesor en el INEA. A este respecto, explicó:

A los quince años, empecé aplicando exámenes y después como tutor. Me gustó mucho la idea de dar clases, me gustó el ambiente, ayudar a las personas a obtener el certificado y enseñé a dos personas a leer y escribir; fue gratificante. Era una oportunidad de enseñar, ya era maestro, las personas en el pueblo me decían maestro. Ahí confirmé mi vocación de ser maestro (participante 7).

Murrieta / Reyes. Experiencias de desempeño y creencias de autoeficacia en profesores de lenguas extranjeras Sinéctica 52 www.sinectica.iteso. $m x$ 
En este caso, observamos un temprano interés de este participante por ser profesor. Es muy probable que en ello haya influido el contexto favorable en que esta experiencia se dio. Se trataba de adultos rurales respetuosos e interesados en aprender a leer y escribir. Estas experiencias positivas precoces parecen haber generado habilidades sicosociales que se reflejaron en estabilidad y satisfacción ocupacional (Bandura, 1994). Efectivamente, este participante eligió la carrera de maestro de lenguas, ha trabajado en diversos contextos, ha impartido una gran variedad de asignaturas, y se ha formado, además, en el ámbito del diseño curricular y las competencias.

Un aspecto adicional que todos los docentes mencionaron como un elemento fundamental para mejorar su eficacia docente fue el esfuerzo. Al inicio de su carrera, todos ellos enfrentaron numerosos problemas derivados de una formación docente deficiente: "Sí, tuve que invertir mucho esfuerzo, pero me sentí mejor en mi clase” (participante 6). Aunque los profesores tuvieron que esforzarse mucho, ellos no creyeron que fuera por baja capacidad; por el contrario, consideraron que mediante el esfuerzo era posible subsanar las deficiencias de su formación inicial y ser más eficaces. Bandura (1997) menciona que el papel del esfuerzo en la autoeficacia difiere según las personas. En el caso de estos profesores, este tuvo efectos positivos, pues les permitió incrementar su percepción de eficacia.

\section{Las experiencias de desempeño como profesores universitarios}

En su faceta como profesores universitarios de tiempo completo, la actividad principal de los participantes es impartir clases de inglés y francés. Podemos decir que, tratándose de clases de lengua, la actividad de los profesores es básicamente la misma que hacían antes de convertirse en profesores universitarios.

No obstante, los participantes también han enfrentado actividades nuevas. Dado que en la Universidad existe una licenciatura para formar profesores de inglés, a excepción de las profesoras de francés, los demás han impartido clases teóricas en inglés en ella. También se cuenta con una maestría en Educación, y casi todos han incursionado en ese nivel en diferente medida. Sin embargo, la experiencia que la mayoría tiene impartiendo clases de lengua es mucho mayor. El participante 7, contrariamente, muestra una fuerte experiencia enseñando materias de contenido en ambos niveles.

Este desfase en la experiencia docente en diferentes tipos de materias ha repercutido en el sentido de autoeficacia que los participantes dicen tener. El participante 7 trabajó de manera simultánea dando clases de lengua y de contenido. Los detalles pueden observarse en la tabla 3 (los números corresponden a años). 
Tabla 3. Tipo de docencia y creencias de autoeficacia

\begin{tabular}{|c|c|c|c|c|c|c|}
\hline P & TDLU & EAU & TDL & AL & TDCLM & AC \\
\hline 1 & 11 & 6.5 & 17.5 & Moderado & 0 & Incapaz \\
\hline 2 & 8 & 6 & 14 & Alto & 6 & Moderado \\
\hline 3 & 16 & 3 & 19 & Alto & 3.5 & Alto \\
\hline 4 & 14 & 5 & 19 & Alto & 1 & Moderado \\
\hline 5 & 14 & 6 & 20 & Moderado & 4.5 & Moderado \\
\hline 6 & 14 & 3 & 17 & Moderado & 4.5 & Moderado \\
\hline 7 & 8 & 15 & 8 & Moderado & 8 & Alto \\
\hline
\end{tabular}

$\mathrm{P}=$ Participante

TDLU= Total de años de docencia en lenguas en la universidad

$\mathrm{EAU}=\mathrm{Años}$ de experiencia antes de la Universidad

$\mathrm{TDL}=$ Total de años de docencia en lenguas

$\mathrm{AL}=$ Autoeficacia lenguas

TDCLM = Total de años de docencia de contenido en licenciatura y maestría

$\mathrm{AC}=$ Autoeficacia contenido

En lo concerniente a las clases de lengua, tres profesoras dijeron tener un alto sentido de autoeficacia sustentado en sus estrategias de enseñanza, la planeación, los materiales empleados y el alto dominio de la lengua y la cultura enseñadas. La participante 4 aseveró: "Tengo la certeza de lo que yo hablo realmente francés y conozco la cultura". Estas docentes tienen ya más de quince años de experiencia y a lo largo de ellos han obtenido el grado de maestría y se han actualizado mediante diversos cursos. Los elementos mencionados han favorecido el desarrollo de un alto sentido de autoeficacia. Bandura (1997) coloca las experiencias reales de desempeño como la fuente más influyente en este tipo de creencias.

Cuatro profesores expresaron creencias de autoeficacia en un nivel moderado. Esta valoración se basó en la falta de dominio de la tecnología para la enseñanza, la poca práctica real del idioma enseñado y problemas en el dominio de aspectos específicos de lengua. Una participante explicó: "Pues, más o menos, es que enseñar inglés en niveles altos es más difícil, es más trabajo" (participante 6). Estos profesores enseñan a universitarios que nacieron rodeados de tecnología, por lo que saber usarla didácticamente es una habilidad insoslayable. En cuanto a la falta de práctica y dificultades de domino de la lengua enseñada, es del conocimiento público que la gran mayoría de profesores mexicanos de lenguas no han vivido o llevado a cabo estancias en el extranjero. En consecuencia, no han tenido oportunidad de mejorar su nivel de idioma, su conocimiento de la cultura y, por ende, su sentido de autoeficacia.

En el caso de estos participantes, los años de experiencia parecen haberlos vuelto más reflexivos y críticos. El identificar sus debilidades puede conducirlos a buscar estrategias de mejora para adaptar su enseñanza; por ejemplo, podrían inscribirse en convocatorias para estancias en el extranjero o participar en seminarios y cursos virtuales para paliar la falta de contextos reales en los cuales practicar el idioma. Bandura asevera que "las dudas sobre uno mismo pueden crear el ímpetu para aprender" (1986, p. 394).

Respecto a las clases en maestría, observamos una mayor variación en las creencias de eficacia docente de los participantes. Únicamente una participante reportó 
un alto nivel tanto impartiendo clases de lengua como de contenido. "Soy bastante buena en lo que hago. Me fue muy bien en la clase de comprensión lectora en inglés de la maestría" (participante 6). El que esta profesora tenga casi veinte años ejerciendo la misma actividad parece haberle permitido extrapolar ese conocimiento a impartir una clase en maestría. Bandura (1997) menciona que algunas actividades generan creencias de autoeficacia muy acotadas, pero que otras pueden percibirse como más generalizables. En este caso, no existe una diferencia importante entre impartir clases de inglés a público en general a impartir clases de comprensión de lectura en inglés en maestría.

Dos de las tres participantes que también habían expresado sentirse muy capaces en clases de lengua dijeron contar con un nivel menor en clases de contenido en maestría: "Me pone muy nerviosa, lo de la maestría. Ahí sí cuestiono mi capacidad y mis habilidades" (participante 4). La otra profesora expresó haberlo hecho moderadamente bien enseñando materias de contenido en maestría, pero destacó que fue gracias a la ayuda de una profesora experimentada: "Sí, gracias a la maestra que estaba antes. Le pedí ayuda. Me dijo: 'No te preocupes yo te voy a dar todo'; me dio su programa, sus materiales y, dijo, si necesitas algo, pregúntame, yo te voy a ayudar" (participante 2).

Es evidente que para estas profesoras enseñar en maestría asignaturas teóricas con contenido complejo les resulta una actividad difícil, porque requieren conocimientos muy distintos a los que por lo regular han enseñado. Se conjugan, entonces, la poca experiencia en estas áreas en particular y el bajo dominio de contenido. El resultado es una valoración de autoeficacia moderada, puesto que la magnitud que los profesores asignan a la tarea es más alta de lo que se consideran capaces (Bandura, 1997). En el caso de la participante 2 , el que haya buscado y recibido ayuda de una colega resultó un aliciente para sentirse capaz de enfrentar esta nueva actividad.

Solo una de las participantes que declaró un nivel moderado de autoeficacia en clases de lengua dijo sentirse incapaz para impartir materias de contenido en licenciatura o posgrado: "Nunca he probado dar clases, bueno, siento que no estoy lista para dar clase en una maestría” (participante 1). Bandura (1997) señala que en las expectativas de eficacia influye la magnitud que la persona asigna a la tarea y que esta, a la vez, repercute en la fuerza que se tenga para emprenderla. En este caso, la profesora no cree que pueda alcanzar un buen desempeño, porque considera que se trata de una actividad muy difícil y no parece tener intención de iniciarse en ella.

Por su parte, las participantes 5 y 6 declararon el mismo nivel de eficacia (moderado) para impartir clases de lengua que de contenido en maestría: "Sí, o sea, porque yo creo que todavía me falta crecer más para seguir dando clases en la maestría, necesito seguirme preparando, me fue más o menos bien" (participante 6). Estos profesores, a pesar de que cuentan ya con muchos años impartiendo clases de lengua y tienen una buena base experiencial en ese tipo de docencia, conciben la profesión docente como algo en lo que hay que estar preparándose de manera constante; por ejemplo, la participante 5 expresó: "Cuando eres profesor te das cuenta de que no sabes nada y que tienes que estar aprendiendo muchas cosas".

Dado que impartir clases en maestría requiere conocimientos y habilidades muy distintas de las que los profesores usualmente han necesitado para impartir clases 
de lengua, parece lógico que estos se perciban, en general, menos eficaces en la primera actividad. La experiencia juega también un papel fundamental. Conviene aquí recordar que, de modo tradicional, el área de lenguas extranjeras se había dedicado a impartir solo clases de idioma. Realizar docencia en licenciatura y, sobre todo, en maestría es una actividad reciente, alrededor de cinco años en el caso de los participantes. Así, los profesores apenas se están ajustando a esta nueva forma de concebir la profesión. Entra en juego aquí la identidad profesional en evolución que repercute en la autoevaluación de eficacia.

El participante 7 fue el único que señaló sentirse con mayor capacidad en clases de contenido que de lengua en ambos niveles: licenciatura y maestría. Explicó: “Me siento más a gusto y más preparado en las clases de contenido en maestría y también en la licenciatura, creo que soy bueno". Este participante abundó: "De hecho, creo que tengo la formación, siento, pienso, creo que tengo la formación didáctica pedagógica para dar clases".

Para Bandura (1997), las creencias de autoeficacia varían en cada dominio de funcionamiento, e incluso, a través de esos dominios, existen gradaciones distintas en las demandas de las actividades o tareas específicas a desarrollar. Debido a que este profesor impartió distintas materias en diversos niveles educativos, adquirió experiencias previas variadas y desarrolló un sentido de autoeficacia más amplio dentro del terreno de la educación. En concordancia con Palmer (2006), también podemos señalar que, además de la experiencia previa, el dominio pedagógico y cognitivo son fuentes de su sentido de autoeficacia.

Como observamos, las creencias de autoeficacia de los participantes varían según la duración de las experiencias y la variedad de contextos en los que se han desempeñado. La actividad que estos profesores han realizado durante más tiempo ha sido la enseñanza de lenguas $y$, a pesar de que al inicio fue una actividad difícil para la mayoría, con el tiempo su sentido de autoeficacia incrementó. Este hallazgo es consistente con la gran mayoría de la literatura sobre el tema (Afshar et al., 2015; Cocca \& Cocca, 2016; Covarrubias y Mendoza, 2015; Klassen \& Chiu, 2010; Morris \& Usher, 2011; Praver, 2014). En cuanto a la variedad de contextos, los resultados son coherentes con el modelo de Tschannen-Moran, Woolfolk y Hoy (1998); es decir, los participantes son conscientes de que impartir clase en otros niveles y otro tipo de asignaturas requiere el despliegue de estrategias y habilidades específicas.

No obstante, cuatro de los participantes no consideran haber logrado aún un nivel alto de eficacia en clases de lengua. Bandura argumenta que las trayectorias de desempeño son variadas e influidas por el contexto, pero lo más importante es la forma en que se procesan las experiencias pasadas. En este caso, los profesores automonitorean selectivamente sus recuerdos y, por ello, difieren en la manera en que recuerdan y evalúan su actuación. Casi todos los profesores parecen haber interpretado sus experiencias negativas como inherentes al proceso de mejora profesional. Hoy recuerdan, en su mayoría, aspectos positivos de su experiencia y estos les permiten contar con creencias de autoeficacia altas o moderadas. La única persona que se declara incapaz de dar clases de maestría es la única que nunca lo ha hecho. Por lo tanto, la falta de experiencia da congruencia a esta autoevaluación. 


\section{ConCLusión}

Esta investigación planteó dos objetivos. El primero fue determinar las experiencias de desempeño que han tenido los profesores a lo largo de su trayectoria y su relación con sus creencias de eficacia docente. Encontramos que los profesores fueron construyendo sus creencias de autoeficacia a partir de una formación que careció de práctica en contextos reales y que influyó en que las primeras experiencias docentes fueran difíciles. No obstante, como profesores noveles, los participantes tuvieron variadas experiencias de desempeño. Algunos de ellos realizaron estancias en el extranjero; quienes lo hicieron durante un periodo largo desarrollaron altas creencias de autoeficacia sobre su dominio del idioma y obtuvieron, además, más experiencia como profesores de lengua que como profesores de contenido.

En su actual trabajo como PTC, los profesores han enfrentado nuevos retos de enseñanza, como impartir clases de contenido en licenciatura y en posgrado. A raíz de ello, encontramos variaciones en las creencias de autoeficacia según se trate de impartir clases de lengua o clases de contenido en licenciatura y posgrado. Existe una variedad de combinaciones a este respecto, solo tres de los siete participantes coinciden en el mismo nivel de autoeficacia en ambos tipos de actividad docente.

En lo anterior influyen varios aspectos. En primer lugar, el propio procesamiento personal de los participantes para leer sus trayectorias. En segundo, el hecho de que seis de los siete participantes obtuvieron una plaza como profesor de tiempo completo a temprana edad (entre los veintiséis y los veintiocho años) y, por ende, se dedicaron casi exclusivamente a impartir cursos de lenguas. Por el contrario, la falta de seguridad laboral llevó al participante 7 a emplearse en niveles y tipos de escuela diversos; este hecho favoreció la construcción de una autoeficacia en contextos más variados, sobre todo en el área de educación.

También es de notar que los profesores vivieron un proceso difícil en la construcción de su autoeficacia. No obstante, para mejorar su enseñanza utilizaron recursos como la reflexión, la toma de cursos extracurriculares, la obtención de grados superiores, el dominio elevado de la lengua que enseñan y el esfuerzo en la planeación de sus clases. Las experiencias de desempeño y el tiempo transcurrido han incrementado las creencias de autoeficacia de los profesores.

Los resultados de este trabajo son consistentes con la gran mayoría de la literatura y con la teoría de la autoeficacia que coloca a las experiencias de desempeño como la fuente más influyente en las creencias de autoeficacia. Gracias a la capacidad de agencia de estos profesores, los obstáculos más grandes fueron sorteados. No obstante, varios de ellos conciben el quehacer del profesor como uno en el cual nunca se termina de aprender, es decir, que con el transcurso de los años se han convertido en profesores más reflexivos y críticos de su propio desempeño; por ello, se autoubican en niveles de autoeficacia moderada.

Estos profesores parecen contribuir a una mejor enseñanza de lenguas dada las características mencionadas. Sus trayectorias pudieran ser ejemplo para futuros profesores en lo que se refiere a la reflexión, el esfuerzo, la formación y el alto nivel de lengua. Aunque, de igual manera, este trabajo muestra la necesidad de que las instituciones implementen estrategias para que los profesores en activo 
puedan, cada determinado tiempo, realizar estancias en países donde se habla la lengua que enseñan y, así, mejorar o actualizar sus habilidades lingüísticas y su sentido de autoeficacia.

Este resultado también invita a hacer hincapié en proporcionar a los profesores en formación experiencias reales de desempeño para generar desde el inicio un buen sentido de autoeficacia. De esa manera, ya como profesores en servicio, pueden realizar una docencia que influya de modo positivo en el aprendizaje de los estudiantes, futuros profesionales en un mundo laboral donde el dominio de las lenguas extranjeras constituye una ventaja.

Esta investigación aporta elementos explicativos para comprender cómo varía el sentido de autoeficacia docente a lo largo del tiempo y sobre los elementos que contribuyen a conformarlo. Estos datos son novedosos dado que se trata de un tema poco explorado en México y en Latinoamérica, sobre todo en lo relacionado con los docentes de lenguas extranjeras. En esta línea de pensamiento se torna importante realizar estudios que investiguen la influencia de las experiencias en el extranjero y la variedad de contextos en la construcción de las creencias de autoeficacia docente, además de investigar cómo estas se reconfiguran al enfrentar nuevos retos. De igual forma, se sugiere explorar cómo la competencia intercultural puede beneficiar la construcción del sentido de autoeficacia docente.

Finalmente, para facilitar el recuerdo de hechos ocurridos hace varios lustros, es deseable que en futuras investigaciones, además del guion, se utilicen durante las entrevistas herramientas de apoyo como fotografías o los currículos. También sería oportuno solicitar a los profesores, por anticipado, una reflexión guiada sobre su trayectoria profesional que permita recordar experiencias docentes y pueda ser utilizada durante la entrevista para estimular las respuestas.

\section{REFERENCIAS BIBLIOGRÁFICAS}

Afshar, H. S., Rahimi, A., Ghonchehpour, A. \& Saedpanah, E. (2015). The impact of teaching experience on Iranian EFL teachers' sense of efficacy and their perception of English teacher distinctive characteristics. Procedia-Social and Behavioral Sciences, núm. 192, pp. 714-719. doi: 10.1016/j.sbspro.2015.06.069

Bandura, A. (1997). Self-efficacy: The exercise of control. Nueva York: Worth $\mathrm{Pu}-$ blishers.

Bandura, A. (1994). Self-efficacy. En V. S. Ramachaudran (ed.). Encyclopedia of human behavior (vol. 4, pp. 71-81). Nueva York: Academic Press.

Bandura, A. (1986). Social foundations of thought and action. Englewood Cliffs, NJ: Prentice-Hall.

Bandura, A. (1977). Self-efficacy: Toward a unifying theory of behavioral change. Psychological Review, vol. 84, núm. 2, pp. 191-215. http://dx.doi. org/10.1037/0033-295X.84.2.191

Best, B. (2014). A study of elementary school Thai English teachers' perceived English proficiency and self-reported English teaching efficacy, Masters Dissertation. Language in India, vol. 14, núm. 7, pp. 1-13.

Brown, J. D. \& Coombe, C. (eds.) (2015). The Cambridge guide to research in Language teaching and learning. UK: Cambridge University Press. 
Cocca, M. \& Cocca, A. (2016). Self-efficacy in pre-school and primary teachers: Does experience, educational background, and teaching level matter? International Conference on Education and New Learning Technologies. Barcelona, España. doi:10.21125/edulearn.2016.0910

Cocca, M., Cocca, A., Alvarado, E. A. \& Rodríguez, M. G. (2018). Correlation between self-efficacy perception and teaching performance: The case of Mexican preschool and primary school teachers. Arab World English Journal, vol. 9, núm. 1. https://dx.doi.org/10.24093/awej/vol9no1.4

Covarrubias, C. y Mendoza, M. (2015). Sentimiento de autoeficacia en una muestra de profesores chilenos desde las perspectivas de género y experiencia. Estudios Pedagógicos, vol. 41, núm. 1. http://dx.doi.org/10.4067/S071807052015000100004

Creswell, J. W. (2007). Qualitative inquiry and research design: Choosing among five approaches (2a. ed.). Thousand Oaks, CA: Sage.

Díez Medrano, J. (2014). The socio-economic returns of fluency in English as a foreign language. En J. Gerhards \& S. Hans (eds.). Globalisierung, Bildung, und GrenzüberschreitendeMobilität (pp. 239-257). Wiesbaden: Springer.

Ghafar Samar, R., Kiany, G. R., Akbari, R. \& Azimi, H. (2011). Institutional identity of teachers: A determinant of teacher efficacy. Teaching English Language, vol. 5, núm. 1, pp. 1-29. Recuperado de http://teljournal.org/en/downloadpaper. php?pid=96\%20\&\%20p=A

Goker, S. D. (2006). Impact of peer coaching on self-efficacy and instructional skills in TEFL teacher education. System, vol. 34, núm. 2, pp. 239-254.

Hernández, R., Fernández-Collado, C. y Baptista, P. (2006). Metodología de la investigación. México: McGraw-Hill.

Hoy, A. W. \& Spero, R. B. (2005). Changes in teacher efficacy during the early years of teaching: A comparison of four measures. Teaching and Teacher Education, vol. 21, núm. 4, pp. 343-356. Recuperado de http://wps.ablongman. com/wps/media/objects/2347/2404137/TATE\%202005\%20Spero.pdf

Klassen, R. \& Chiu, M. M. (2010). Effects of teachers' self-efficacy and job satisfaction: Teacher gender, years of experience, and job stress. Journal of Educational Psychology, núm. 102, pp. 741-756. http://dx.doi.org/10.1037/ a0019237

Lengeling, M. M. (2010). Becoming an English teacher: Participants' voices and identities in an in-service teacher training course in central Mexico. Guanajuato, México: Universidad de Guanajuato.

Marashi. H. \& Azizi-Nassab, F. (2018). EFL teachers' language proficiency, classroom management, and self-efficacy. International Journal of Foreign Language Teaching \& Research, vol. 6, núm. 22, pp. 89-102. Recuperado de http:// jfl.iaun.ac.ir7/article_601313_8f68bb32b9b726999f2c5262903fc9ec.pdf

Mirsanjari, Z., Karbalaei., A. \& Afraz, S. (2013). Teacher' sense of efficacy in teaching English among Iranian EFL teachers. Indian Journal of Fundamental and Applied Life Sciences, vol. 3, núm. 3, pp. 417-428. Recuperado de http:// www.cibtech.org/J-LIFE-SCIENCES/PUBLICATIONS/2013/Vol_3_No_3/JLS53-61-ALIREZA-TEACHERS-TEACHERS.pdf

Morris, D. B. \& Usher, E. L. (2011). Developing teaching self-efficacy in research institutions: A study of award-winning professors. Contemporary Education Psychology, vol. 36, núm. 3, pp. 232-245. http://dx.doi.org/10.1016/j.cedpsych.2010.10.005 
O’Donoghue, L. J. (2015). Sorry. El aprendizaje del inglés en México. México: Mexicanos Primero. Recuperado de http://www.mexicanosprimero.org/index. $\mathrm{php} /$ educacion-en-mexico/como-esta-la-educacion/estado-de-la-educacion-en-mexico/sorry-2015

Palmer, D. (2006). Sources of self-efficacy in a Science methods course for primary teacher education students. Research in Science Education, vol. 36, núm. 4, pp. 337-353. doi: 10.1007/s11165-005-9007-0

Praver, M. (2014). Japanese university English language teachers' self-efficacy beliefs: A mixed-methods exploration. Tesis doctoral. Recuperado de http:// digital.library.temple.edu/cdm/ref/collection/p245801coll10/id/280496

Reyes-Cruz, M. R. y Murrieta-Loyo, G. (2017). Los futuros profesores de inglés: experiencia previa y sentido de autoreficacia. MEXTESOL Journal, vol. 41, núm. 2, pp. 1-17. Recuperado de http://mextesol.net/journal/public/files/2 6dbb63dcecf38bd592d3630122aff39.pdf

Secretaría de Educación Pública (2008). La enseñanza de idiomas en México. Diagnóstico, avances y desafíos. DGAR. Reunión Nacional de Control Escolar. Documento interno.

Singh, K. (2007). Quantitative social research methods. Thousand Oaks, CA: Sage.

Slami, Z. \& Fatahi, A. (2008). Teachers' sense of self-efficacy, English proficiency and instructional strategies: A study of nonnative teachers EFL teachers in Iran. TESL-EJ, vol. 11, núm. 4. Recuperado de http://tesl-ej.org/ej44/a1.html

Sun, S., Chen, H. \& Song, Z. (2016). Cross-level moderating effects of conscientiousness on within-person relationships of self-efficacy to effort allocation. Human Performance, vol. 29, núm. 5, pp. 447-459. https://doi.org/10.1080/ 08959285.2016 .1245733

Tschannen-Moran, M. \& Hoy, A. W. (2007). The differential antecedents of selfefficacy beliefs of novice and experienced teachers. Teaching and Teacher Education, vol. 23, pp. 944-956. doi:10.1016/j.tate.2006.05.003

Tschannen-Moran, M. \& Hoy, A. W. (2001). Teacher efficacy: Capturing an elusive construct. Teaching and teacher education, vol. 17, núm. 7, pp. 783-805. Recuperado de https://pdfs.semanticscholar.org/42e3/af5061de43d25b85 144964ab7a3e44a7549f.pdf

Tschannen-Moran, M., Woolfolk, A. \& Hoy, A. W. (1998). Teacher efficacy: Its meaning and measure. Review of Educational Research, vol. 68, núm. 2, pp. 202-248. Recuperado de http://wmpeople.wm.edu/asset/index/mxtsch/ rer1998 